\title{
FACTORIZATION OF HYPONORMAL OPERATORS
}

\author{
MARY R. EMBRY \\ (Received 29 November 1969) \\ Communicated by E. Strzelecki
}

In [1] R. G. Douglas proved that if $A$ and $B$ are continuous linear operators on a Hilbert space $X$, the following three conditions are equivalent:

(i) $A(X) \subset B(X)$;

(ii) $A A^{*} \ll \lambda B B^{*}$ for some $\lambda \geqq 0$;

(iii) there exists a continuous linear operator $C$ on $X$ such that $A=B C$. It was also shown that the operator $C$ is unique within the constraints

a) $\|C\|^{2}=\inf \left\{u \mid u \geqq 0\right.$ and $\left.A A^{*} \ll u B B^{*}\right\}$,

b) kernel $C=$ kernel $A$, and

c) $C(X) \subset \overline{B^{*}(X)}$.

The purpose of this note is to consider the special case in which $B=A^{*}$; that is, $A(X) \subset A^{*}(X)$.

Definition. $\mathscr{D}$ is the set of all continuous linear operators on $X$ such that $A(X) \subset A^{*}(X)$. If $A \in \mathscr{D}, C_{A}$ is the unique operator such that $A=A^{*} C_{A}$,

$$
\left\|C_{A}\right\|^{2}=\inf \left\{u \mid u \geqq 0 \text { and } A A^{*}=u A^{*} A\right\},
$$

kernel $C_{A}=$ kernel $A$, and $C_{A}(X) \subset \overline{A(X)}$.

It is immediate from Douglas' theorem that every hyponormal operator $A\left(A A^{*} \ll A^{*} A\right)$ is an element of $\mathscr{D}$. In this note we characterize the hyponormal operators as those elements of $\mathscr{D}$ such that $\left\|C_{A}\right\| \leqq 1$. A normal operator $A$ is characterized as an element of $\mathscr{D}$ such that $C_{A}$ is a partial isometry with $C_{A}(X)=$ $\overline{A(X)}$ and a self-adjoint operator $A$ is characterized as an element of $\mathscr{D}$ for which $C_{A}$ is the projection on $\overline{A(X)}$.

In this paper $A(X)$ denotes the range of $A$ and kernel $A$ denotes the null space of $A$. I denotes the identity operator on the Hilbert space $X$. We repeat the following definition of and facts concerning partial isometries, found in [2, p. 99].

1) $U$ is a partial isometry if and only if $U$ is an isometry on the orthogonal complement of kernel $U$;

2) the range of a partial isometry is closed; 
3) if $U$ is a partial isometry, $U^{*} U$ is the projection on (kernel $\left.U\right)^{\perp}$;

4) the adjoint of a partial isometry $U$ is a partial isometry; and

5) $U$ is a partial isometry if and only if $U=U U^{*} U$.

THEOREM 1. Let $A \in \mathscr{D} . A$ is hyponormal if and only if $\left\|C_{A}\right\| \leqq 1$.

Proof. If $\left\|C_{A}\right\| \leqq 1,\left\|A^{*} x\right\|=\left\|C_{A}^{*} A x\right\| \leqq\|A x\|$ for all $x$ in $X$ and $A$ is hyponormal. Assume now that $\boldsymbol{A}$ is hyponormal. Since

$$
\left\|C_{A}^{*} A x\right\|=\left\|A^{*} x\right\| \leqq\|A x\| \text { for all } x \text { in } X,
$$

$\left\|C_{A}^{*} y\right\| \leqq\|y\|$ for all $y$ in $\overline{A(X)}$. Let $P$ be the projection on $\overline{A(X)}$ and note that $C_{A}^{*}(I-P)=0$ since $C_{A}(X) \subset \overline{A(X)}$. Thus for each $x$ in $X$,

$$
\left\|C_{A}^{*} x\right\|=\left\|C_{A}^{*} P x\right\| \leqq\|P x\| \leqq\|x\|
$$

and consequently, $\left\|C_{A}\right\|=\left\|C_{A}^{*}\right\| \leqq 1$.

THEOREM 2. Let $A \in \mathscr{D} . A$ is normal if and only if $C_{A}$ is a partial isometry with $C_{A}(X)=\overline{A(X)}$.

Proof. If $C_{A}$ is a partial isometry and $C_{A}(X)=\overline{A(X)}$, then $C_{A}^{*}$ is an isometry on $\overline{A(X)}$. Therefore,

$$
\left\|A^{*} x\right\|=\left\|C_{A}^{*} A x\right\|=\|A x\|
$$

for all $x$ in $X$ and $A$ is normal. Assume now that $A$ is normal. Then

$$
\|A x\|=\left\|A^{*} x\right\|=\left\|C_{A}^{*} A x\right\|
$$

for all $x$ in $X$ and $C_{A}^{*}$ is an isometry on $\overline{A(X)}$. Since

$$
\overline{A(X)} \supset \overline{C_{A}(X)}=\left(\text { kernel } C_{A}^{*}\right)^{\perp},
$$

$C_{A}^{*}$ is a partial isometry. Therefore $C_{A}$ is a partial isometry and $C_{A}(X)$ is closed. It is easy to show that $C_{A}(X)=\overline{A(X)}$, using the facts that $C_{A}(X)$ is a closed subset of $A(X), A=A^{*} C_{A}$ and $A$ is normal.

In view of the fact that $C_{A}$ is a partial isometry if $A$ is normal it is reasonable to ask when $C_{A}$ is an isometry and when $C_{A}$ is unitary. With one restriction $\left(C_{A}(X)=\overline{A(X)}\right)$ these are both equivalent to $A$ being normal and one-to-one. This is seen as follows. If $A$ is normal and one-to-one, $C_{A}$ is (by Theorem 2) a partial isometry with kernel $C_{A}=\{0\}$ and $C_{A}(X)=\overline{A(X)}=X$. Thus $C_{A}$ is an isometry whose range is all of $X$ and hence is unitary. Conversely, if $A \in \mathscr{D}$ and $C_{A}$ is an isometry with $C_{A}(X)=\overline{A(X)}, A$ is normal (by Theorem 2) and one-toone (since kernel $A=$ kernel $C_{A}=\{0\}$ ). The restriction that $C_{A}(X)=\overline{A(X)}$ is necessary in this equivalence, for if $A$ is an isometry, then by the criteria for uniqueness it can be shown that $C_{A}=A^{2}$. There exist many examples of nonnormal isometries and thus many isometries $C_{A}$ associated with non-normal operators $A$. 
COROLlary 1. If $A$ is normal, then $C_{A}$ is normal.

Proof. By Theorem 2, $C_{A}$ is a partial isometry with $C_{A}(X)=\overline{A(X)}$ if $A$ is normal. Therefore $C_{A}^{*} C_{A}$ is the projection on $C_{A}^{*}(X)$ and $C_{A} C_{A}^{*}$ is the projection on $C_{A}(X)$. However, since $A$ is normal,

$$
C_{A}(X)=\overline{A(X)}=\overline{A^{*}(X)}=\left(\text { kernel } C_{A}\right)^{\perp}=C_{A}^{*}(X)
$$

and therefore $C_{A} C_{A}^{*}=C_{A}^{*} C_{A}$.

The converse of Corollary 1 is not valid as is seen by the following simple example: if $A=\left(\begin{array}{rr}1 & i \\ 0 & -1\end{array}\right)$, then $A \in \mathscr{D}$ and $C_{A}=\left(\begin{array}{rr}1 & i \\ -i & 2\end{array}\right)$, which is normal. More generally, if $A^{2}=I$ as in this example, then $C_{A}=A^{*} A$.

Characterizations of normal operators different from that found in Theorem 2 can be obtained as corollaries of Theorem 2 .

CoROllaRY 2. Let $A \in \mathscr{D}$. The following conditions are equivalent:

(i) $A$ is normal;

(ii) $A C_{A} C_{A}^{*}=A$;

(iii) $A$ commutes with $C_{A}^{*}$.

Proof. ( $\Rightarrow$ ii). If $A$ is normal, then by Theorem 2 and Corollary $1 C_{A}$ is a normal partial isometry. Therefore

$$
A C_{A} C_{A}^{*}=A C_{A}^{*} C_{A}=\left(A^{*} C_{A}\right) C_{A}^{*} C_{A}=A^{*} C_{A}=A .
$$

(ii) $\Rightarrow$ (iii). If (ii) holds, $C_{A}^{*}$ is an isometry on $\overline{A^{*}(X)}$. Since

$$
A \in \mathscr{D}, \overline{A^{*}(X)} \supset \overline{A(X)} \supset \overline{C_{A}(X)}=\left(\text { kernel } C_{A}^{*}\right)^{\perp}
$$

and thus $C_{A}^{*}$ and $C_{A}$ are partial isometries. Moreover,

$$
A(X) \subset A^{*}(X) \subset C_{A} C_{A}^{*} A^{*}(X) \subset C_{A}(X) .
$$

Therefore, since $\left(C_{A} C_{A}^{*}-I\right) C_{A}=0,\left(C_{A} C_{A}^{*}-I\right) A=0$. This last equation yields $A=C_{A} C_{A}^{*} A=C_{A} A^{*}$. Since by definition $A=A^{*} C_{A}$, we see that $A^{*} C_{A}=C_{A} A^{*}$ and that $A$ commutes with $C_{A}^{*}$.

(iii) $\Rightarrow$ (i). Assume that $A C_{A}^{*}=C_{A}^{*} A$. Then

$$
A A^{*}=\left(A^{*} C_{A}\right) A^{*}=A^{*}\left(A^{*} C_{A}\right)=A^{*} A
$$

by definition of $C_{A}$ and we see that $A$ is normal.

Corollary 3. Let $A \in \mathscr{D} . A$ is self-adjoint if and only if $C_{A}$ is the projection

Proof. If $A$ is self-adjoint, then $A^{*}\left(C_{A}-I\right)=0$, and by Theorem $2, C_{A}(X)=$ $\overline{A(X)}$. It follows that $\left(C_{A}^{*}-I\right) C_{A}=0$ and thus that $C_{A}$ is the projection on $\overline{A(X)}$. 
Assume now that $C_{A}$ is the projection on $\overline{A(X)}: C_{A} A=A$. Then $A^{*}=A^{*} C_{A}^{*}=$ $A^{*} C_{A}=A$ and $A$ is self-adjoint.

The last theorem in this note is a characterization of normal partial isometries.

THEOREM 3. $C$ is a normal partial isometry if and only if there exists a normal operator $A$ such that $C=C_{A}$.

Proof. By Theorem 2 and Corollary 1 we know that if $C=C_{A}$ ( $A$ normal), then $C$ is a normal partial isometry. On the other hand if $C$ is a normal partial isometry and $A$ is any normal square root of $C$, it can be shown that

$$
A=A A^{*} A=A^{*} A^{2}=A^{*} C,
$$

$A(X)=C(X)$ and kernel $A=$ kernel $C$. Thus $C=C_{A}$ if $A$ is any normal square root of $C$.

\section{References}

[1] R. G. Douglas, 'On majorization, factorization, and range inclusion of operators on Hilbert space', Proc. Amer. Math. Soc. 17 (1966), 413-415.

[2] P. R. Halmos, A Hilbert Space Problem Book (Van Nostrand, Princeton, N.J. 1967).

University of North Carolina at Charlotte 